\title{
MEGACOLON EN EL NIÑO. SU TRATAMIENTO ACTUAL
}

Drs. GUILLFRMO ZIEGLER S. y J,UIS FUENTES DE LA S.

Clínica Universitaria de Citugla Infantil y Ortopedia del Prof. Arnulfo Johow. Hospital "Roberto del Rio"

En el año 1886 fuć descrita por Hirschs- pertrofia de la última porción del intestino prung una entidad anátomo-clínica caracteriza- grueso y un recto de aspecto macroscóticicamenda por retención estercorácea, dilatación e hi- te normal. Esta enfermedacl era congénita y no 
obedecia a ninguna causa mecánica de obstrucción, liué denominada Megacolon Congénito o Enfermedad de Frirschsprung. Su tratamiento era sintomatico, médico-dietético y quinúrgico. La cirugia hasta hace pocos años resecaba las partes dilatadas del colon o hacia simpatecto mías. Los resultados obtenidos con estos tra- cstudiados, Whitehouse y Kernohan demostraron el porcentaje de ausencia de los plexos de Auerbach y Meisner en los diferentes segmentos del intestino terminal.

La figura $\mathrm{N} 2$ muestra los porcentajes en que estos plexos mientéricos faltaban en los casos de Whitehouse y Kernohan en los dife-

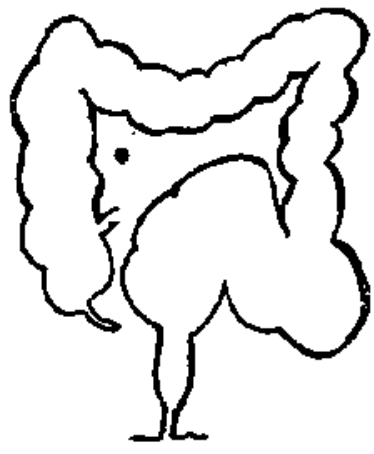

A

Figura No $1: A-M e$. gacolon congénito: o verdadera Enfermedad de Hirschsprung, con una olsstrucción funcional del recto por contracciones no impulsivas (achalnsicas) y una enorme dila. tación de la $\mathrm{S}$. signoidea $y$ a veces dicl colon, por encima cle esta obstrucción. lil recto en estos casos es racliológicamente de calibre nomal.

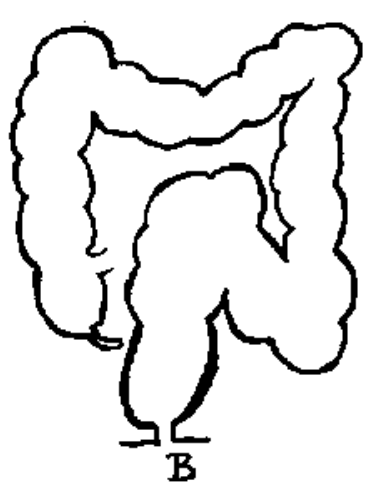

B. Megacoton idiotodti. co.-En este grupo nosotros agrupanos los du. licosigmas, dolicomegasig. mas y demás megacolones de causa funcional sin achalasia rectal. En radiología es frecuente encontrar dilataciones dc diversos segmentos cólicos sin un gran megacolón acompañante.

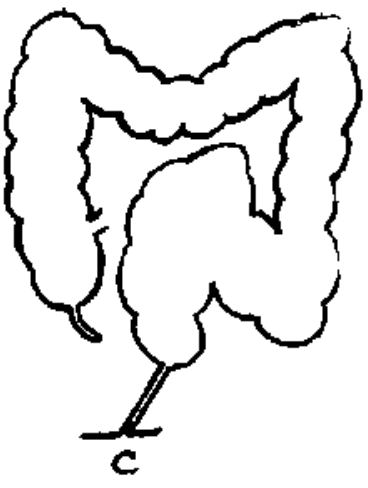

C. Megacolon sintoma. tico.- Consecueucia de malformaciones anales o ano-rectales, producidas por lo tanto por una obs. trucción mecínica. Lo más frecuente en el mi. ño es la interforacion anal, con fistula insuficiente. tamientos eran francamente malos en nuestra clínica, como en todlas las utras del mundo.

Lnicamente en los últimos años con el mejor conacimiento de su patogenia, se ha podido diferenciar anátomo-clínica y radiológicamente 3 tipos de megacolon.

1. La patogenia exacta del megacolon congénito fué demostrada por estudios histopatológicos practicados por Whitehouse y Kernohan (5), aunque nuchos alutores como Haw. kins (1), Alvarez (2), Hurst (3), Bockus (4) sospechaban, ya hace años la causa de esta enfermedad: un defecto neuromuscular por el cual el intestino es incapaz de evacuar su contenido sin existir una causa mecánica. En 11 casos de Megacolon Congénito cuidadosamente rentes segmentos del recto y $\mathrm{S}$ sigmoidea. Posteriormente otros autores han descrito achalasias que se extendian a todo el colon hasta la válvula ileocecal y un caso en que la ausencia de plexos mientéricos llegaba hasta el ángulo de Treitz, comprometiendo en este caso la casi totalidad del intestino grueso y delgado.

Los estudios anátomo-patológicos del Dr. Gurmán (anátomo-patológo de nuestro hospital) demostraron igualmente ausencia de dichos plexos de Auerbach en todos nuestros casos operados a nivel del recto, presentando la $\mathbf{S}$ sigmoidea muchos menos plexos que una $\mathbf{S}$ normal.

En el intestino normal, las fibras nerviosas parasimpáticas terminan en los plexos de Auer. bach y por medio de éstos provocan la con- 


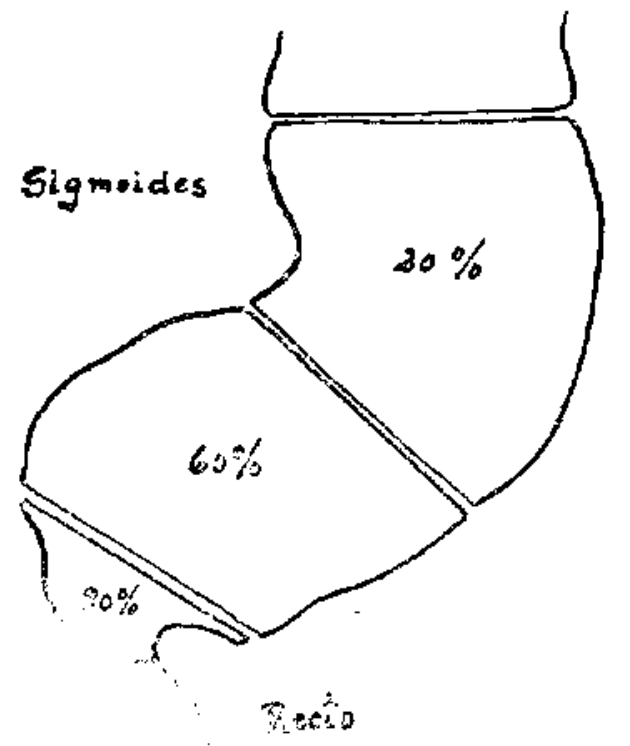

traccion peristáltica capaz de hacer avanzar el contenido. En los intestinos disquinéticos, achalásicos (sin plexos de Aucrbach) los impulsos parasimpáticos producen contracciones en masi, que no tienen propictades impulsivas; se constituye asi una obstrucción funcional, cuya consecuenciál es la dilatación e hipertrofia del intestino que esti por encima cle csta achalasia, para vencerla.

2. Se desconoce lin verdadera patogcnia del megacolon idiopático. Idiopático significa de causa desconocidia. Para la mavoría de los autores delyen intervenir varios factores.

Según hodian, existiria un megarrecto por una estrechez funcional del ano. Nosotros creemos que las molestias en esios casos de negadolicosigyma y megarrecto congénitos, se producen por la gran superlicic de absorción de agua que facilita la formación de fecalomas, que posteriormente producen obstrucción intestinal. De aquí que el tratamiento en estos casos puede ser médico dietético, el cual consiste en evitar la formación cle fecalomas y apurar el tránsito intestinal.

3. La patogenia del megacolon sintomático u orgánico es mecánica: tumor, trauma o es- trechez anorectal, con el adulto $y$ mallormaciones anorrectales congtnitas, en el niño. Fl tratamiento del megacolon congénito sintomático sería la corrección guirúgica de la malformación anorrectal, facilmente diagnosticable, como en el caso de la lotografía atdunta (Figura No 9$)$.

Micniras que el dinguóstico de megacolon sintomatico se hace por el examen físico clel entermo, cl diagnóstico exacto de megacolon congénio o idiopático y la dilerenciación de ambos, se hice exclusivamente por al estudio radiológico. Fs importante difcrenciar ambos megacólones de causa funcianal, pues son de diferente pronóstico y tratamiento. Actualmente gracias a los estudios de Neuhauser podemos alemostrar por medio de la xadiología la región achalásica o espástica, la cual obrando como obstruccion incompleta prodace la dilatación

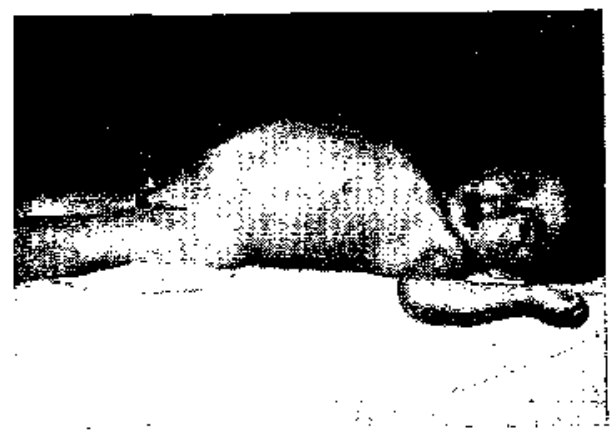

Figstia No 3: Niño con megacolon sintomitico a una iraperforación anal, sxte nitho defe. caba por una fístula anterior insuficiente. Operándolo de su malformacion anorrectal sanó de sal megacolon.

e hipertootia de los segmentos si:uados inmediatamente por encima de esa zoma (Véanse Figuras N.os 4 y 5 ).

Hiatt (12) considera que no hay diferencia patogénica entre estos dos tipos de megacolon funcional descritos y que los casos con megarectos obedecen a una achalasia anal, que a veces no requicre cratamiento quirúrgico. Este autor usa, para ambos tipos de megacolon, la misma operación de Swenson. 


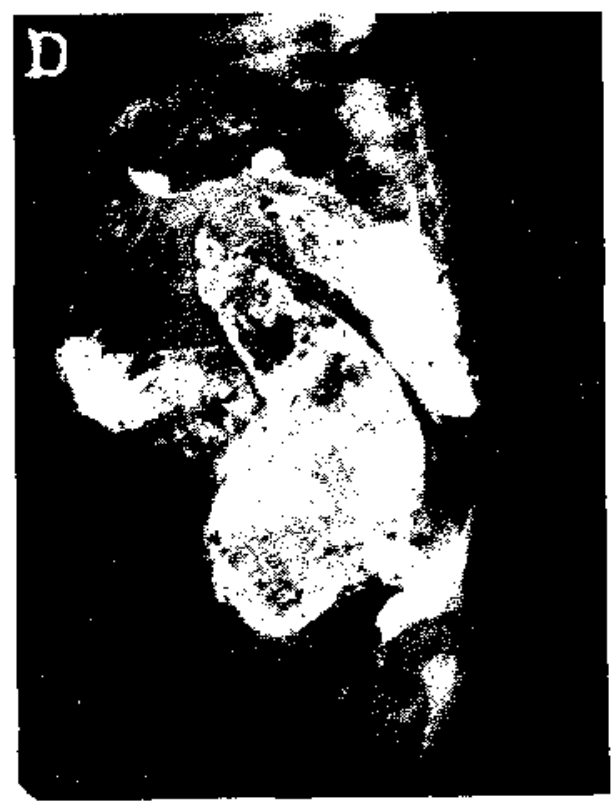

Highere Ne t: Radiografía de un megateolon conyenito, también llatiado por Hurst y Bockus megacolon por achalasja del recto y por Bodian. Stephers y Ward, verdadera crlf. ale Hirchsprung.

s. caracteriza por constipación que sc inicia desde el nacimiento. P'rácticamettte el enfermo sólo defeca dyulado por enemas y sifomajes. Consecucincia de esto es la ¿ilatación e lipertrofia de.] colon rlistal. que va cxtendicindose hasta comprometerlo en su totalidad. Fenómenos de olutriteción inlcstinal frecuentes por raticnción cstetcolécea. Mortalictad alta por obstrucción intestimal aguda por vólvutos. Recto normal al tacto y al examen rectoscópico.

El ryotha baritado especialmbente en ln posirim oblinta demuestra fue la dilota. ción comienza cu forma més o mevos brtsca por encima det recter $v$ este conserva nut calibse nommatl. FI diagúsósion cxácto k.

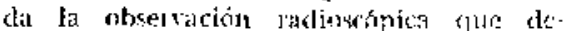
numesta la faita cle rontracciones isoperistílticas rel recto por ausencia de sus plexos de Auerbach y Meisster.

Tratamients: Es superfluo repet ir que el tratamier to del megacolon sintomático a una malformación anorrectal es quirúrgico: operar la malformación anorrectal.

Jin cuanu al megatcolon idiopático con megarrecto o dolicomcgasigna juede mejorar con un tratamiento médico-dictético bicn llevado. Tenemos cuatro casos nuestros en los cuales se ensayó el tratamiento médico-dietético con éxito, tratamiento que bay que mantener durinte muchos años, evitúndose con esto una operación grave. Esto no sucede en el megacolon congćnito, debido a una achalasia del recto. Para contirmar la inutilidad del cratamiento médico-dietćtico en los megacólones congénitos, todos nuestro operados fueron tratados previamonte duranie meses en el Servicio de Medicina de nuestro Hospital médicn-dietéticamente, ensayándose dilerentes medicamentos, sifonajes y regímenes. I.legamos a la misma conclusión de otros autores de que este grupo de los megacolones congénitos no nejoran con tratamiento médico, ni con resecciones de los segmentos dilatados o simpatectomias, hechas también infruccuosamente en nuestra clinica en otra época.

Sólo la rectosigmoidectomía abdominaperineal con conservación det estinter anal, ideada por Swenson (6) perinitc la curación del megacolon congénito. Corresponde a nuestro prolesor A. Johow luaberla introducido en Chile, operación que reseca en primer lugar la parte achalísica del recto y en segundo lugar el máximo del intestino dilacado.

En cuanto al megacolon idiopático, solamente se operan atquellos casos en que el tratamiento médico-dietético bien llevado fracasa o cn casos que por su condición social no pueden cumplir dicho tratamiento. La operación puede ser la misma de Swensan o una mucho mís sencilla: una simple sigmoidectomía, que es la preferida por nosotros, es decir, la cxtirfación de los segmentos dilatidos, impidiendo con csto gue el estancaniento de las materias fecales $y$ la exageradi absorción de agua de las deposiciones produza la lormación de tecalomas. Consideramos que depende deI grado de la dilatacion del recto la decisión de practicar una u otra operación.

No estamos de acuerdo con Hiatt que efectúa indiferentemente lát operación de Swenson en atubos casos, puesto que esta speración demora 5 horas en tanto que una colectontía sólo 2 horats, con mucho menos riesgo operatorio. En el prcoperatorio son importantes los sifonajes, los cuales deberán ser bechos con suero fisiológico o agua bicarbonatada y no con agua pura, la cual, por ser hipotónica, 


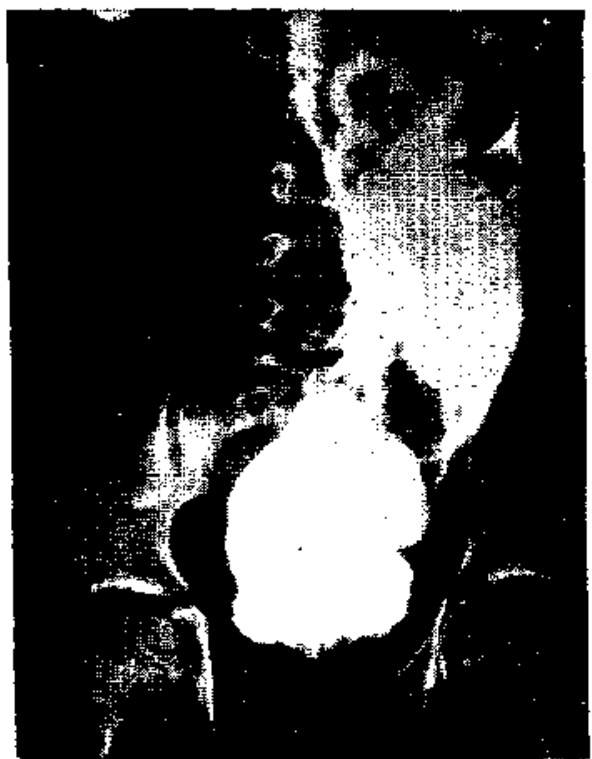

Figura No 5: Radiografía de un megacolun islioprition a megarresto sin arhala. sia anal.

Màs frecuente que al meatolon congenito: tiene sut origen en una constipación crónica que se inicia en general tardía. mente, mucho ulespućs del nacimiento.

Varios factores etiológicos han sido invocalos: un eslínter anal espástico, nial hitbito de cvacuacion de las deposiciones. um colon sigmoideo congénitamente más largo. que provoca exagerada absorción de agua de las deposiciones o una achalasia anal. T.n general son varios los factores en jucgo.

Es de pronóstico más benigno que el megacolon congónito. Unicamente el ene. ma harilado lo diferencia del megacolon con achalasia rectal demostrando una dilatasion que se ritiende desde el mismo conducto anal, in recto y una $s$. sinmoiden dilatada y contuncciones peristicts. (Es importante anotar que el conducto anal en el nîño presenta un largo de 2 a $3 \mathrm{~cm}$. y es preciso no confundirlo cot un recto achalásico).

Los plexos mientéricos son normales. lin radiologia es frecuente encontrar dilataciones de diversos segmentos intestimales sin ningena sintomtatología clínica, In doli. cosigma puede ser un hallazgo radiológico. Pero tarde o temprano estos niños pueden ser candidatos a hacer un megacolon con gran sintomatología, como nuestros casos operados únicamenté despues de haber cnsayado infrucluositmente el trat. mćlicoclictético.

puetle producir graves estados de shock al bajar la concentración del sodio sanguineo.

I a operatción de Swenson yue practicamos actualmerte es la modilicada por Hiatt, cuyos detalles fueron publicados en los Anales de Cirugía, Vol. 19, N⿳3, píg. 376 , en marzo de I951. El fundamento de esta intervención es la resección del segmento disquinético, achalísico, por vía perineal, produciendo un prolapso artificial, que facilita la operación y evita la contaminación de la cavidad abdominal.

Para producir dicho prolapso es necesario disecar previamente el recto-sigmoides por via abdomúnal, lacilitando con esto su movilización. Prolapsado el intesino, se secciona y se suturan circularmente los calbos restantes en dos planos a unos clos coms. por encima del límite mucosa-piel. Conseguida la continuidad del intestino por esia sutura, se invagina nuevamente para adentro la anastómosis, la cual

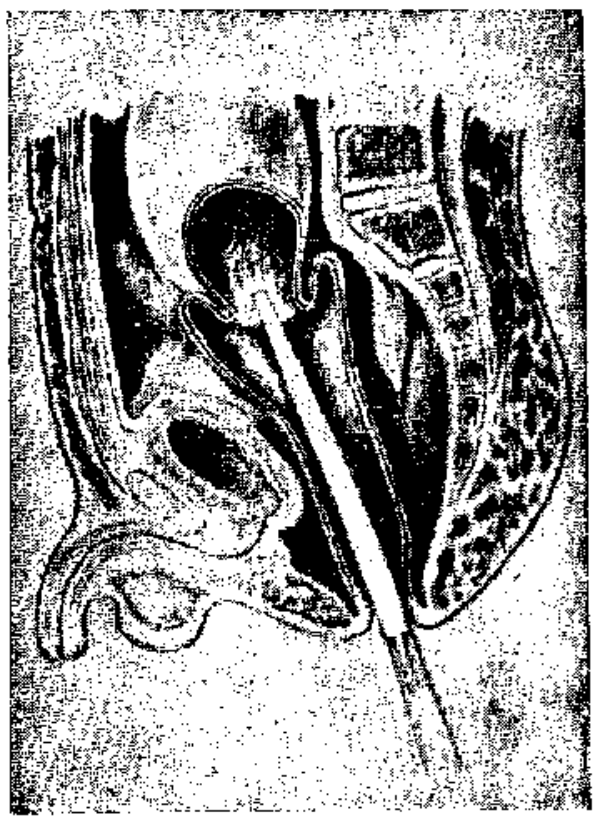

FIK TURA NI G

queda mantenida per el esfinter anal conser. vado e intacto (Vianse Figuras N.cs 6,7 y 8 ). La operacien de Swenson la hemos practicado siempre en un tiempo. Algunos autores la efectúan en des tiempos con un ano contranatura provisorio previo, para excluir la zona operatoria del trínsito intestinal. Nosotros, en Ia Clinica del protesor 1 . Johow, no conside- 
ramcs necesario esto, puesto que un bucn examen y una bueria preparación preoperatoria del entermo con sifonajes 2 veces al dia, regi men especial, antibióticos, ftalilsulfacetimida oral y transfusiones, nos permite operar con

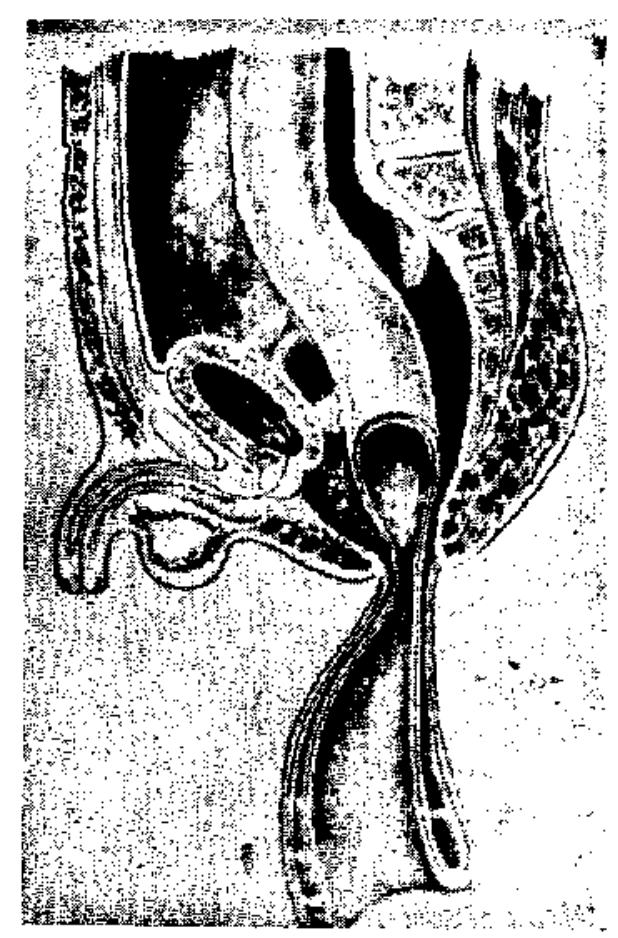

ligi:RA Yo 7

intestino limpio, casi asćptico, en un enlemo con relativo buen estado general.

Con el primer enfermo operado hemos aprendido que no basta resecar solamente la parte disquinćticá, es decir, el recto-sigmoides achalásico. Hay que resecar además la mayor parte del scgmento dilatado, dejando un trayecto recto entre el ángulo coloes jelénico y el conducto anal. En el primer enfermo operado resecamos únicamente el recto disquinético transformando con esto el megacolon congénito en uno idiopitico, que nos obligó posteriormente a electuar una nueva resecrión por vía abdominal, esta ve\% con bnen resultado.

Esta operación no produce incontinencia del esfinter anal. Ĺl colon llevado a la región perineal en operaciones que conservan el esfínter anal, es capaz al distenderse de pro- ducir cierto grado de sensación de tipo rectal con la consiguiente contracción del estínter anal. Se explica esto (Coligher) (11) por la distenciún de las estructuras pélvicas. Por lo tanto para obtener un ano continente es necesario hacer la disección del recto pegado a su adventicia, yara eviar dañar las estructuras pélricas responsables de las sensatciones rectales.

En una enferina se produjo clurante la divulsión anal, para prolapsar el intestino, un desgarro de la mucosa y del esfinter anal, que se complicó con una pelviperitonitis postoperatoria y muerte de la enferna dos meses despućs a consecuencias de una peritonitis plástica postoperatoria. Consideramos actualmente que esta enferina no se hubiera muerto si se le hubiera practicado un ano contra natura de đlescirga, inmediatimente después de la operación.

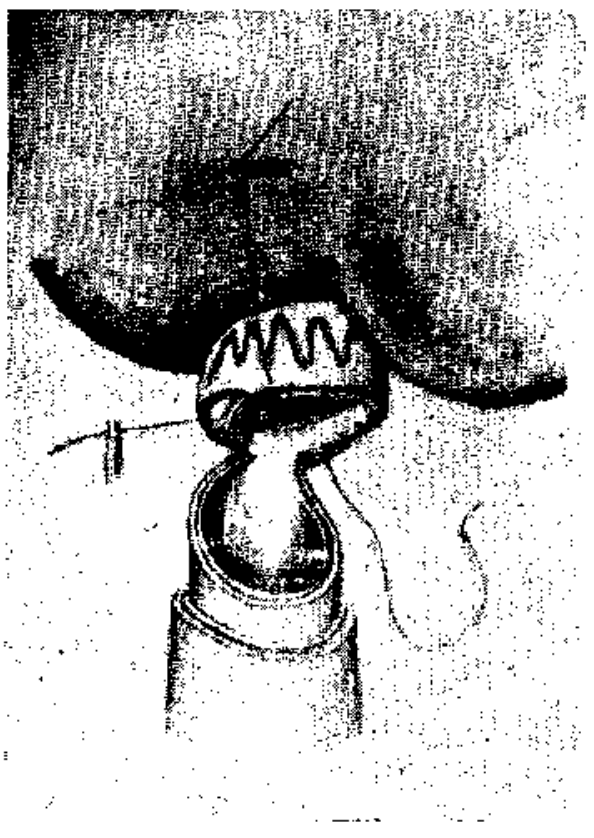

IIGUIR.A NO 8
Analizamos en el cuadro los resultados alejados de las intervenciones quirúrgicas efec. tuadas, con controles, el más antiguo, dos años después de la operación. Todos los megacólo nes sintomáticos operados de su malformación 
anorrectal han quedado en perfectas condiciones, regresando su megacolon.

Hemos operado 5 megrarólones jdiopáticos. Otros, cuatro respondicron al tratamiento mćdico-dietćtico bien llevado. En elles efectua- ciones diarias espontíneas, continencia deI esIinter anal y reducción progresiva del tamaño del abdomen. Los inemas baritados de control clemuestrin una paulatina reducción de la dilatación del colon.

CUADRO NO 1

\begin{tabular}{|c|c|c|c|c|c|c|c|c|c|c|c|c|c|c|}
\hline \multirow[b]{2}{*}{$\begin{array}{l}0 \\
\not R \\
=\end{array}$} & \multirow[b]{2}{*}{ 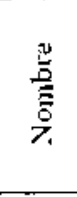 } & \multirow[b]{2}{*}{ 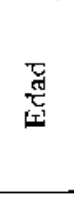 } & \multirow[b]{2}{*}{ 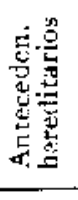 } & \multirow[b]{2}{*}{ 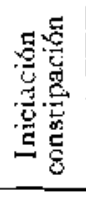 } & \multirow{2}{*}{\multicolumn{2}{|c|}{ 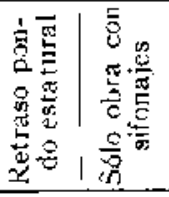 }} & \multirow[b]{2}{*}{ 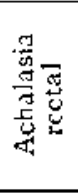 } & \multirow[b]{2}{*}{ 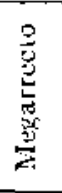 } & \multirow[b]{2}{*}{ 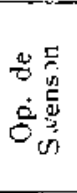 } & \multirow[b]{2}{*}{ 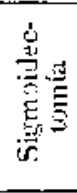 } & \multicolumn{3}{|c|}{ CONTROLES } & \multirow[b]{2}{*}{ 䓪 } \\
\hline & & & & & & & & & & & $\begin{array}{l}5 \\
0 \\
0 \\
5 \\
5 \\
5\end{array}$ & 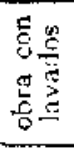 & 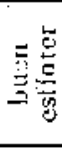 & \\
\hline \multicolumn{15}{|c|}{ Mcgacelon crngenito } \\
\hline $\begin{array}{l}1 \\
2 \\
3 \\
4 \\
5 \\
6 \\
7 \\
8\end{array}$ & $\begin{array}{l}\mathrm{CB} \\
\mathrm{FA} \\
\mathrm{SS} \\
\mathrm{DSF} \\
\mathrm{RA} \\
\mathrm{MM} \\
\mathrm{CP} \\
\mathrm{JL}\end{array}$ & $\begin{array}{c}8 \mathrm{81} \\
4 \mathrm{a} \\
12 \mathrm{a} \\
11 \mathrm{In} \\
11 \mathrm{a} \\
5 \mathrm{a} \\
2 \mathrm{a} \\
2 \mathrm{a}\end{array}$ & + & $\begin{array}{l}\text { nasc. } \\
\text { nasc. } \\
\text { nasc. } \\
\text { nass. } \\
\text { nasc, } \\
\text { nasc. } \\
\text { nase. } \\
\text { nasc. }\end{array}$ & $\begin{array}{l}+ \\
+ \\
+ \\
+ \\
+ \\
+ \\
+ \\
+\end{array}$ & $\begin{array}{l}+ \\
+ \\
+ \\
+ \\
+ \\
+ \\
+ \\
+\end{array}$ & $\begin{array}{l}+ \\
+ \\
+ \\
+ \\
+ \\
+ \\
+ \\
+\end{array}$ & $\begin{array}{l} \pm \\
\pm\end{array}$ & $\begin{array}{l}+ \\
+ \\
+ \\
+ \\
+ \\
+ \\
+ \\
+ \\
+\end{array}$ & & $\begin{array}{l}+ \\
+ \\
+ \\
+ \\
+\end{array}$ & + & $\begin{array}{l}+ \\
+ \\
+ \\
+ \\
+ \\
+ \\
+\end{array}$ & + \\
\hline \multicolumn{15}{|c|}{ Mogacolon idiopático } \\
\hline $\begin{array}{l}1 \\
2 \\
3 \\
4 \\
5\end{array}$ & $\begin{array}{l}M R \\
\text { AC } \\
\text { ED } \\
\text { LA } \\
\text { EM }\end{array}$ & $\begin{array}{r}8 \mathrm{a} \\
5 \mathrm{a} \\
4 \mathrm{a} \\
13 \mathrm{a} \\
12 \mathrm{a}\end{array}$ & & $\begin{array}{l}5 a \\
3 a \\
1 a \\
4 a \\
8111\end{array}$ & $\begin{array}{l}+ \\
+ \\
+ \\
+\end{array}$ & $\begin{array}{l}+ \\
+ \\
+ \\
+ \\
+\end{array}$ & & $\begin{array}{l}+ \\
+ \\
+ \\
+\end{array}$ & & $\begin{array}{l}+ \\
+ \\
+ \\
+ \\
+\end{array}$ & $\begin{array}{l}+ \\
+ \\
+ \\
+ \\
+\end{array}$ & & $\begin{array}{l}+ \\
+ \\
+ \\
+ \\
+\end{array}$ & \\
\hline
\end{tabular}

mos la resección de la $S$ sigmoidea y en un caso además resecamos el colon descendente. La sutura terminoterminal se hizo según técnica de Braun que permite suturar trozos de intestino de distinto calibre. En todos los casos obtuvimos la mejoría rlinica y radiológica de la enfermedad.

En cuanto a los megacólones por achalasia rectal hemos operado ocho según la técnica de Swenson y variantes.

El diagnóstico clínico-radiológico se comprobó con el extudio histopatológico de las piezas operatorias extraídas.

En el primer caso operacto tuvinos que reintervenir dos meses clespués. por resección insuficiente, quedando el enfermo bien deșués de una nueva resección más amplia.

Podemos decir, por to tanto, que de 8 enfermos operados 6 quedaron bien, con deposi-
Insistimos en que durante el postoperatorio habrá que hacer la reeducación de los músculos abdominales, los cuales, por el abombamiento abdominal, están distendiclos y sin huerza para ayudar a la defecación. Además deberá evitarse a toda costa la retención estercorácea, por medio de la vaselina líquida oral para conseguir la reducción del calibre del colon restante, insisticndo a los enfermos en que evacuen su intestino puntualmente durante un largo período. In apuellos casos en que no contamos con la cooperación familiar, es preferible dejarlos hospitalizados un largo ticmpo, evitutudo así las recaidas por falta de cumplimiento de las indicaciones dadas.

Resumen: Se reconienda como único tratamiento racional del megacolon congénito la operación de Swenson moditicada por Hiatt. En cambio, en el megacolon idioprítico convie- 
ne ensayar el tratamiento médico antes de pensar en la intervención quirúrgica, que puede ser la misma operación de Swenson o la simple resección de la $S$ sigmoidea dilatada, según el caso. Estas intervenciones pueden ser cjecutadas a cualquier edad.

\section{SUMMARY}

As the only rational treatment for congenital megacolon, Swenson's operation modified by Hiatt is recommended. On the other hand, in the idiopatic megacolon medical treatinent should be tried before thinking of a surgical intervention, which can be the same Swenson's operation or a simple resection of the dilated S. sigmoid depending on the case. These operations can be performen at atry age.

\section{B I B L I O C R A F I A}

1.-Hawkins. H. P.: "Remarks on Icliopathic I ilatation of the Colon". Brit. M. J. 1 (477), 1907.
2.-Alvaret, W C.: "An Introduction to Gastro-Enterology". New York, Paul B. Hnelor, Ine., 1940.

3.-Ilurst. A. F.: "Anal Achalasia and Megacolon (Hirschsprung's Disease Idliopathic Dilatiotion of the Colon)". Giy's Hosp. Rep. 84 (\$17). 1(194.

4.-Bockus, H. L.: "Gastro Enteroloms:" Phitadtiphia, W. 13. Saunders Company, 194.3.

5.-Whitehotse, F. and Kernolıan. J. W.: "Myenteric lexus in Congenital Megacolon". Arth. Intern. Med. 82 (75) 1.948.

6.-Bodian, M., Ste'jhens, F, D., alue Ward, B. C: 11.: "Flirschsprung's Discase and Icliopalhis Megacislon". Lancet. 25I (b), 1949.

7.-Swinson, O., antl Bill. A. H., Ji.: "Resction of Rectum and Rectosignoid with preservation of the sphincter fot lening spastic lisions protucing Megacolon". Surgery, 24 (212). 1948.

e.-Lec, M. C.. Jr. et at. "The Selectice Management of Megacolon in Infants and Chikdren". Siarg. Gyn.. and Obst., 91 (281), 1950 .

9.-Swenson, O+ Newheluser, Li, B, D., and liak:tt. L. K. "New comopts of the ctiology. diagnos:s and treatment of congenital megacolin íHitschsprung's (lise:se)". Pccliatrics, 4 (201), 1049.

10.-Bodian, M. Cater, C. O., and Watd, B. C. H.: "Herschsptung's Diseass". Lance, 260 (302), 195 I.

11.-Coligher, Y. C., and Hughes, R. S, R.: "Sensibility of the Rectum and Colon". Ianct, 260) (343), $19.5 \mathrm{I}$.

12.-Hiatı, R. V.. Anales de Cirngia, 10 (376), 1951. 